\title{
Study of the application of solar energy on the air-conditioned train in Qinghai-Tibet Railway
}

\author{
WANG Ye ${ }^{1,2}$, YAN Ruowen ${ }^{1}$ \\ ${ }^{1}$ School of Environmental and Municipal Engineering, Lanzhou Jiaotong University, Lanzhou, \\ 730070, China \\ ${ }^{2}$ Key Laboratory of Railway Vehicle Thermal Engineering, Ministry of Education of China, Lanzhou \\ Jiaotong University, Lanzhou, 730070, China \\ E-mail: wangye@mail.Izjtu.cn
}

Keywords: Qinghai-Tibet Railway; solar radiation; air-conditioned train; solar energy application Abstract. To study the contribution of the solar energy resource to reducing energy consumption of air-conditioned train running along the Qinghai-Tibet Railway, the No. z6811 air-conditioned train of $25 \mathrm{~T}$ was chosen as the research object and the intensity of the solar radiation on the outer surfaces of the train's enclosure was calculated. On the basis of the calculation of the solar radiant intensity, the top outer surface was chosen to be the reasonable position to install the solar panels because of its biggest solar radiant intensity and the longest radiating time and the working temperature, efficiency, power and electricity of the solar panels were calculated and analyzed respectively in winter and summer along the railway. The results show that in summer, the average efficiency of the solar cells of one-way train (Golmud to Lhasa) is $19.08 \%$, the average power is $2.158 \mathrm{~W}$ and the total generated energy is $352.436 \mathrm{kWh}$. In winter, the average efficiency of the solar cells of one-way train (Golmud to Lhasa) is $21.18 \%$, the average power is $1.435 \mathrm{~W}$ and the total generated energy is $224.782 \mathrm{kWh}$. The work in this paper provides an idea for the study of the integration between solar energy and the plateau train.

\section{Introduction}

As the most important and clean energy, solar energy has been widely applied to the industrial, transportation and other fields and makes a lot of research results [1,2].The Qinghai-Tibet plateau is high altitude areas of west China with rich solar energy resources [3]. When Qinghai-Tibet Railway air-conditioned train go through the high altitudes, its ancillary equipment power falls sharply. This phenomenon makes the train operation energy consumption be higher than that of the train running in plain area. How to make use of the abundant solar energy resources to reduce energy consumption of train running along the Qinghai-Tibet Railway is an important approach for reducing the train running cost. At present, the research on the application of solar energy on train has been reported rarely. Based on the air-conditioned train of 25T's intensity of the solar radiation, statistical computing environment temperature of station and solar panels working temperature, the one-way train (Golmud to Lhasa) battery efficiency, power and power generation's curve are obtained.

\section{Physical Model}

The physical model is shown in Fig. 1. Face 1 and 2 are the vertical planes on both sides of the outer surfaces of the train respectively. Face 3 is the top and face 4 is the bottom. 


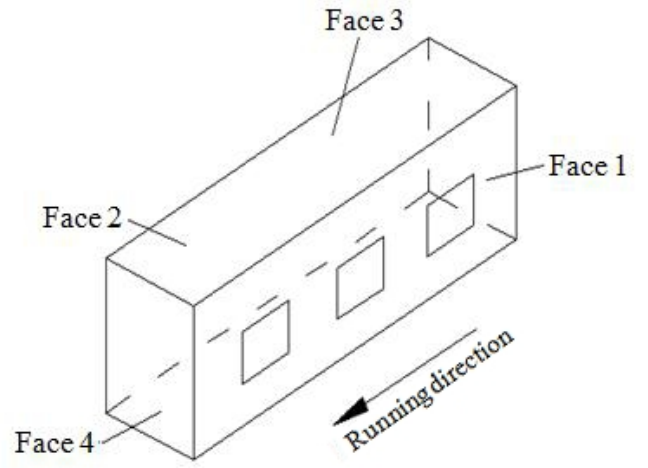

Fig. 1 Physical model

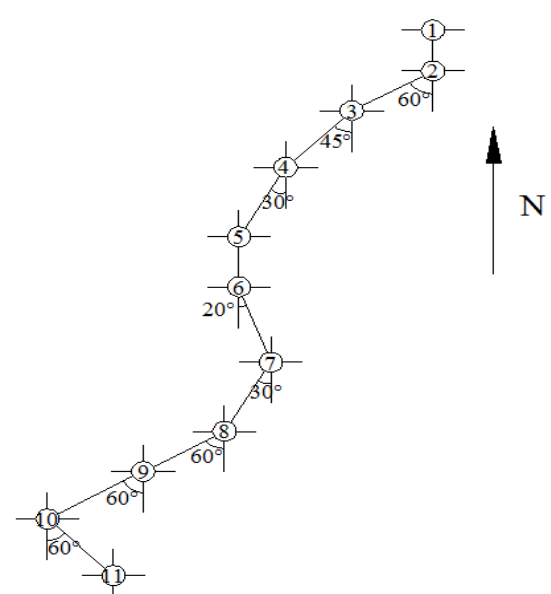

Fig. 2 Train orientation diagram

The position parameters are calculated to receive solar radiation direct intensity and solar radiation scattering intensity. The curve about solar total radiation intensity of the train is obtained. The train orientation diagram is shown in Fig. 2.

Under the condition of the battery temperature $T$, the solar cell maximum power $P_{\mathrm{T}}$, power $P$, working temperature $T$, thermal equilibrium relationship and the battery efficiency are determined by the following formula, respectively.

$$
\begin{gathered}
P_{\mathrm{T}}=\left[1+(T-25) \alpha^{\prime}\right] P_{\mathrm{S}} \\
P=I_{\mathrm{Z}} \times P_{\mathrm{T}} / 1000 \\
\varepsilon I \mathrm{Z}(1-\varphi)=\varepsilon \sigma\left(T^{4}-T_{\text {sky }}{ }^{4}\right)+h\left(T-T_{\mathrm{a}}\right) \\
\varphi=P_{\mathrm{T}} /(1000 \times A)
\end{gathered}
$$

\section{Results and Discussion}

The total intensity of the solar radiation curve of the outer surface of the studied train in summer is shown in Fig. 3 (a). It can be seen that all the surfaces can receive the sun radiation after station 2. Face 1 receives total radiation intensity which is bounded by station 6 with large amplitude. And the maximum value near the station 5 is about $800 \mathrm{~W} / \mathrm{m}^{2}$. For the solar altitude and the wall-sun azimuth, the solar radiation received by face 1 is increasing while the solar radiation on face 2 is decreasing. The fluctuation of the total radiation intensity curve of face 4 is small. It changes within the range of 0 $\sim 216.04 \mathrm{~W} / \mathrm{m}^{2}$ and obtains the maximum value of $216.04 \mathrm{~W} / \mathrm{m}^{2}$ near the station 7 . The intensity of the solar radiation received by face 3 is increasing and then falling down quickly, which has reached the maximum value about $1080.22 \mathrm{~W} / \mathrm{m}^{2}$ near the station 7 . Undoubtedly, the solar radiation absorbed by face 3 is the most abundant The total intensity of the solar radiation curve of the outer surface in winter is shown in Fig. 3 (b). According to the relevant parameters changing with time, every surface begins to receive solar radiation only after station 4 . The change about the sun radiation intensity shows the similar characteristics in summer. The intensity of solar radiation on face 1 near the station 5 and 7 get the maximum value $600 \mathrm{~W} / \mathrm{m}^{2}$. The intensity of solar radiation on station 2 is rising fast and then falling suddenly and after station 8 its fluctuation is small. Face 4 begins to receive solar radiation since station 4 which is rising slowly. After the maximum value on station 7 , it slows down.

Whenever in summer or winter, the variation of the intensity of the solar radiation along the outer surface of the train reflects the changing direction of the train and the solar altitude. In a word, how to maximize the solar energy utilization is related to the schedule of train's operation and solar energy utilization equipment[4]. 


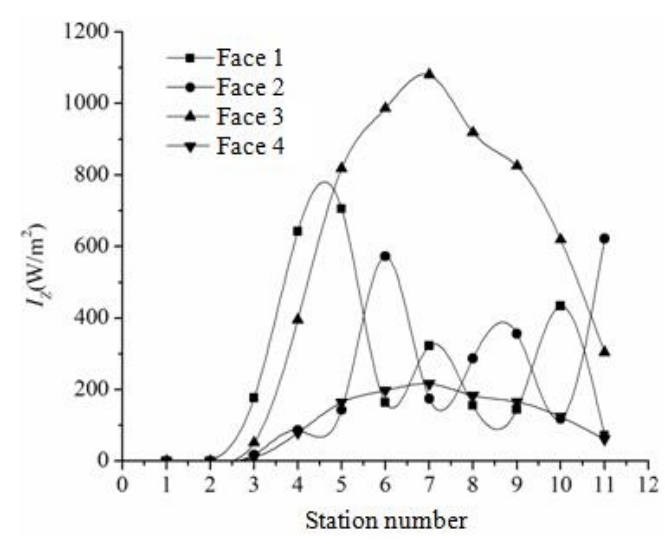

(a)Summer

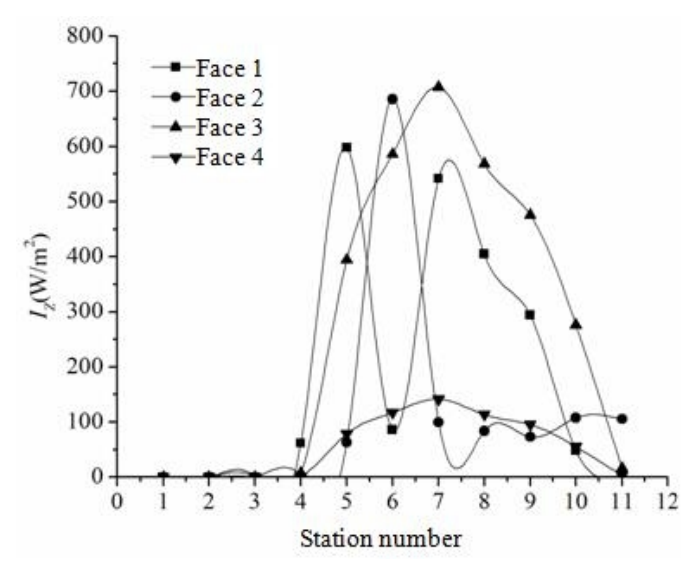

(b) Winter

Fig. 3 Intensity of the solar radiation

\section{Solar energy utilization}

According to the above analysis, whenever in winter or summer, the total intensity of the solar radiation of face 1 has the longest time and good continuity. Therefore, the most appropriate location of the solar panels should be at the top of the train. The solar panel substrate's good heat insulation performance also can effectively reduce energy consumption of the body.

The dimension of face 3 is $L \times W=25500 \mathrm{~mm} \times 3100 \mathrm{~mm}$. Face 3 is covered is covered by 5040 HH125M165-S2 single crystalline silicon solar cell and each photovoltaic module includes 144 crystalline silicon solar cell. On standard condition, HH125M165-S2 single crystalline silicon solar cell's power is $P_{\mathrm{S}}=2.833 \mathrm{~W}$, the efficient is $\varphi=18.2 \%$ and the maximum power temperature coefficient is $\alpha^{\prime}=-0.46 \% /{ }^{\circ} \mathrm{C}$. Train speed is assumed to be the average value according to the speed of adjacent stations.

Fig.4 presents the solar cell temperature curve obtained by Eq.(3). Either in summer or winter, during the running time, the value of solar battery temperature has the similar tendency along the railway. It gets the maximum value on station 8 . This inspires people how to make full use of high temperature to save energy consumption of train. The result can provide a theoretical reference for train running energy saving strategy.

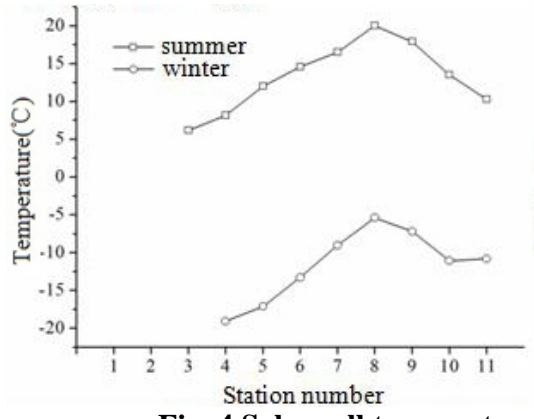

Fig. 4 Solar cell temperature

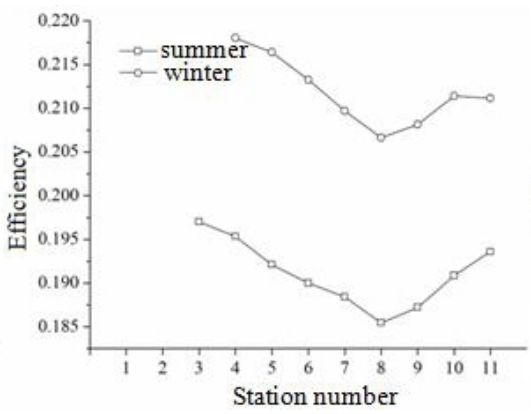

Fig. 5 Solar cell efficiency

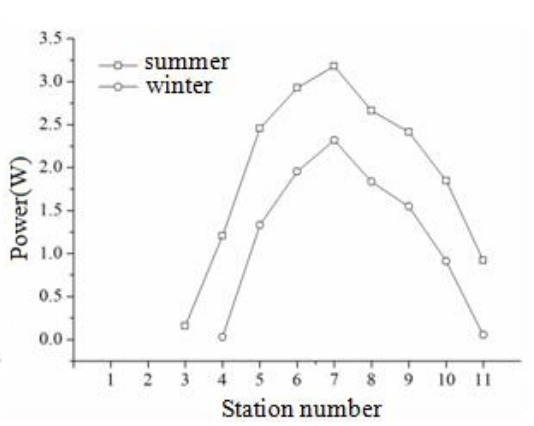

Fig. 6 Solar cell power

Fig.5 presents the battery efficiency curve obtained by Eq. (1) and Eq. (4). It can be seen that the battery efficiency trend is opposite to the battery temperature tendency. The utilization efficiency of solar energy in winter is higher than that in summer.

Fig.6 presents the battery power curve obtained by Eq. (2). It can be seen that the curve of the battery power is similar to the solar total radiation intensity curve. The value of solar battery power in summer is higher than the corresponding period in winter. In summer, the solar cell average efficiency of the one-way train is $19.08 \%$, the average power is $2.158 \mathrm{~W}$ and single carriage solar panels generating capacity is $23.496 \mathrm{kWh}$ of 15 carriages. In the case of installed solar panels, the total generating capacity is $352.436 \mathrm{kWh}$. In winter, the corresponding data are $21.18 \%, 1.435 \mathrm{~W}$, $14.985 \mathrm{KWh}$ respectively. And the total generating capacity is $224.782 \mathrm{kWh}$. 


\section{Conclusions}

The intensity of the solar radiation of z6811 along the Qinghai-Tibet Railway train in different seasons was calculated. The parameters such as solar energy utilization efficiency were obtained in this paper. Some useful conclusions are obtained as follows.

(1) The solar radiation intensity received by every surface varies greatly during the running time. The radiation intensity on face 1 and 2 has the similar tendency in summer and winter. It is suitable to locate a solar panel on the top surface because of the highest intensity of solar radiation and longest radiation time.

(2) The solar cell temperature curve is opposite to the efficiency curve trend while the battery power is similar to the top's total radiation intensity curve. The total generated energy of the solar cells of one-way train (Golmud to Lhasa) is $352.436 \mathrm{kWh}$ in summer while the data is $224.782 \mathrm{kWh}$ in winter.

(3) The solar power - train integrated energy saving operation mode is built by using the abundant solar energy resources of the Qinghai-Tibet plateau. In the part of the time, as the an effective supplement to the train energy, it has important social and practical significance to reduce fuel consumption and protect the ecological environment along the Qinghai-Tibet Railway.

\section{Acknowledgements}

This research was financially supported by the National Natural Science Foundations of China (No. 51476073 and No.51266004) the Gansu Natural Science Foundation (1308RJZA151) and the Gansu construction project of science and technology(JK2016-2).

\section{References}

[1] Zhu Xiongfeng, Guo Zheng, Hou Zhongxi. Solar-powered airplanes: A historical perspective and future challenges[J]. Progress in Aerospace Sciences, 2014, 71: 36-53.

[2] Gao Xianzhong, Hou Zhongxi, Guo Zheng, et al. Reviews of methods to extract and store energy for solar-powered aircraft[J]. Renewable and Sustainable Energy Reviews, 2015, 44: 96-108.

[3] Chen Youyu, Yao Lanchang, Wang Wenhua. The characteristics of surface radiation and its annual variation in Nagqu area of Qinghai-Xizang Plateau[J]. Plateau Meteorology, 1985, 4(4): $50-66$.

[4] Xu Ran, Wang Jingwen, Wang Ye. Study on the relationship between the train running timetable of Qinghai-Tibet Railway and the solar radiation[J]. Journal of Lanzhou Jiaotong University, 2013, 32(6): 165-170. 